Check for updates

Cite this: New J. Chem., 2021, 45, 3833

\title{
Exploring alkaline routes for production of TEOS-based consolidants for carbonate stones using amine catalysts
}

\author{
Alexandra Rodrigues, (D)*a Bruno Sena da Fonseca, (D) ${ }^{b}$ Ana Paula Ferreira Pinto, \\ Susana Piçarra a and M. Fátima Montemor ${ }^{b}$
}

\begin{abstract}
This work aims at developing TEOS-based sols for the consolidation of carbonate stones, following basic catalysis routes, which have been scarcely explored. The influence of the alkaline media in the sol-gel chemistry is a well-known phenomenon and has been considered a drawback. This work demonstrates that under controlled and well-selected conditions, there is high potential on this novel approach and important advantages. Basic catalysts containing $\mathrm{NH}$-goups (ammonia, $n$-octylamine, diethanolamine and triethanolamine) were used in the production of TEOS-based sols. The role of the different amines on the consolidation efficacy of the final gels was then investigated. The development of new TEOS-based solutions with potential consolidation efficacy has been achieved by tailoring several parameters (e.g. precursor/catalyst and precursor/solvent ratios). Optimisation of parameters and modification of the silica structure with different amines allowed us to produce a set of consolidants for carbonate stones. The morphology and structure of xerogels were characterised, sol behaviour was studied after their blending with calcite powder, as well as after their application on soft limestone samples. Cohesive monoliths of calcite powder were obtained, and significant and uniform drilling resistance increments in treated stones were achieved using sols with different TEOS : ethanol : catalyst ratios.
\end{abstract}

Received 21st September 2020 Accepted 20th November 2020 DOI: 10.1039/d0nj04677a

rsc.li/njc limitations that have been reported over time. Besides the typical tendency to crack during the drying-shrinking phase,${ }^{10,11}$ the lack of strong chemical interactions (affinity) between the carbonate minerals and the consolidating material, ${ }^{1,6,7}$ and the general negative effect of the basic media promoted by the substrate itself in the sol-gel routes ${ }^{1,12}$ have been widely reported as the main drawbacks. These limitations have justified numerous studies to develop new products many by modifying and improving the alkoxysilane performance - with potential to serve as carbonate-stone consolidants (e.g. Sena da Fonseca et al. ${ }^{13}$ ).

Functional alkoxysilanes follow the sol-gel process and the conditions on which hydrolysis and condensation reactions occur - which depend on parameters such as $\mathrm{pH}$, and type and amount of solvent catalyst, amongst others - are critical for the performance of the final material. Commercial products often include neutral catalysts, such as dibutyltindilaurate (DBTL) or equivalent organotin compounds, but unfortunately they present reproductive, carcinogenic and mutagenic toxicities that are currently well known and are considered the main reasons to abandon such products. ${ }^{14-16}$ Additionally, these neutral catalysts seem to introduce delayed hydrolysis reactions, which are responsible for hydrophobicity of the consolidated surface, with unwanted consequences. ${ }^{17}$ Finally, 
the lack of scientific understanding of some sol-gel paths and role of some by-products (lauric acid) in these reactions and on the final network, compared to other types of catalysts, also raises some knowledge gaps. ${ }^{16,18}$

Acidic and basic catalysts, and the nature of the catalyst itself, are determinants for the final gel morphology, as such parameters exert great influence on the rate and extent of hydrolysis, that in some cases might be faster and more complete than in the presence of neutral catalysts. The resulting xerogel is actually determined by the type of catalyst. ${ }^{1}$ Acid catalysed TEOS-based sols have recently been reported as potential successful consolidant alternatives for application on carbonate-based stone materials (e.g. Sena da Fonseca et al. ${ }^{5}$ ).

Nonetheless, due to the inherent basicity of carbonate stones, the catalytic activity of most common catalysts is reduced once the formulation is applied onto these stones (e.g. Salazar-Hernández et al. ${ }^{15}$ Mosquera $e t$ al. ${ }^{19}$ and Sena da Fonsec ${ }^{20}$ ) since the sol-gel paths are modified in a way that the final silicate structure may display poor capacity to provide cohesion. ${ }^{12}$ Therefore, the use of base-catalysed consolidants for carbonate stones appears as an alternative to overcome such drawbacks, despite the fact that they have not yet been properly explored.

It is known that base-catalysed hydrolysis and condensation reactions of TEOS tend to produce nanoparticles (instead of the characteristic tridimensional networks that originate in the presence of acid catalysts). However, the formation of these porous silica nanoparticles can potentially decrease the contraction of the xerogel or even reduce its cracking tendency, providing that self-assembly occurs. ${ }^{5,21}$ Alkoxysilane alkaline additives containing $\mathrm{NH}$ groups have recently been studied, using several types of approaches - catalysis, templating, and grafting (e.g. Sena Da Fonseca et al., ${ }^{5}$ Sena da Fonseca et al.., ${ }^{13}$ Mosquera et al., ${ }^{19}$ Sena da Fonseca et al., ${ }^{20}$ Illescas and Mosquera, ${ }^{22}$ and Xu et al. ${ }^{23,24}$ ). Alkanolamines, such as diethanoolamine and triethanolamine (DEOA, and TEOA), have been used as possible templating agents for the formation of mesopores in TEOS-based silica structures. ${ }^{25}$ The $\mathrm{NH}$ groups in amines can act as basic catalysts, rendering the corresponding conjugated acids and $\mathrm{OH}^{-}$anions. ${ }^{5,25}$ Amines still have other effects in the overall sol-gel kinetics, being involved in nucleation mechanisms, templating, or acting as surfactants, as is the case of $n$-octylamine already studied for conservation materials. ${ }^{19,26}$ However, the behaviour of the NH-groups of organic basic catalysts in the final performance depends on several undisclosed variables; hence their role is not yet fully understood. ${ }^{5}$
The purpose of the present work is to explore the influence of different basic catalysts on the production of TEOS-based sols, aiming at consolidating carbonate stone materials. The sols studied in this work were produced using four different basic catalysts containing NH-groups: ammonia, $n$-octylamine as a primary amine $\left(\mathrm{RNH}_{2}\right)$, DEOA as a secondary amine $\left(\mathrm{R}_{2} \mathrm{NH}\right)$ and TEOA as a tertiary amine $\left(\mathrm{R}_{3} \mathrm{~N}\right)$. DEOA and TEOA also possess two and three ${ }^{-} \mathrm{OH}$ terminal groups, respectively (see Fig. 1). The results provide an insight into the roles that these different amines play in the formation of the silica network using TEOS as a precursor. The potential efficacy of the developed sols, as consolidants for carbonate stones, is investigated and discussed.

\section{Experimental}

\subsection{Materials}

The reagents used for the formulation of the sols were: tetraethyl orthosilicate (TEOS) as a precursor, ethanol at 96\% (EtOH) as a solvent, and distilled water for the hydrolysis reactions. As basic catalysts (i) ammonia solution $25 \%\left(\mathrm{NH}_{3}\right)$, (ii) octylamine (Oct), (iii) diethanolamine (DEOA) and (iv) triethanolamine (TEOA) were used. The calcium carbonate powder used was a precipitated salt with $96 \% \mathrm{CaCO}_{3}$, a finely ground product tested according to Ph Eur. All reagents were purchased from Sigma-Aldrich and used without further purification.

Carbonate stone samples used for testing the sols were "Ançã stone" a very homogeneous and soft limestone (relevant monumental building and sculpture stone employed in the Portuguese territory). Ançã stone has an accessible porosity of around $27 \%$, a coefficient of water absorption by capillarity of around $150 \mathrm{~g} \mathrm{~m}^{-2} \mathrm{~s}^{0.5}$ and a pore-size range between 0.1 and $1 \mu \mathrm{m}$, as reported in previous applications. ${ }^{2,12,13}$

\subsection{Preparation of sols and trials}

The sols were prepared by mixing TEOS with EtOH and stirring for 30 minutes, followed by the addition of distilled water and one of the basic catalysts, also under magnetic stirring $(\approx 500 \mathrm{rpm})$ for another 30 minutes in a closed flask at room temperature $\left(24 \pm 2{ }^{\circ} \mathrm{C}\right)$ - following a procedure similar to the one reported by Xiang et al. ${ }^{27}$ Three different molar ratios of TEOS:EtOH $(1: 38,1: 7.6$, and $1: 3.8)$ were used. Whilst the highest dilution (TEOS: EtOH ratio fixed at $1: 38$ ) resulted well as a coating, ${ }^{27}$ lower dilution of TEOS have proved to be more adequate for stone consolidation (e.g. Sena da Fonseca et al. ${ }^{13,20}$ ). Each catalyst was used in different TEOS:(Cat) molar ratios

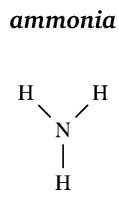

Fig. 1 n-octylamine

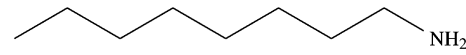

$\mathrm{HO}$

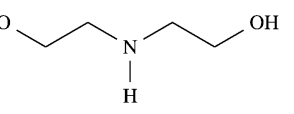

TEOA

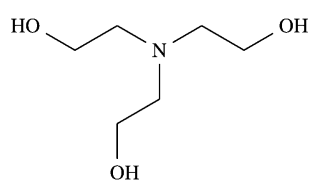


Table 1 Molar ratios used for the preparation of sols, and their relation with the sols' final designations ((Cat) representing the different catalysts applied: $\mathrm{NH}_{3}$, Oct, DEOA or TEOA)

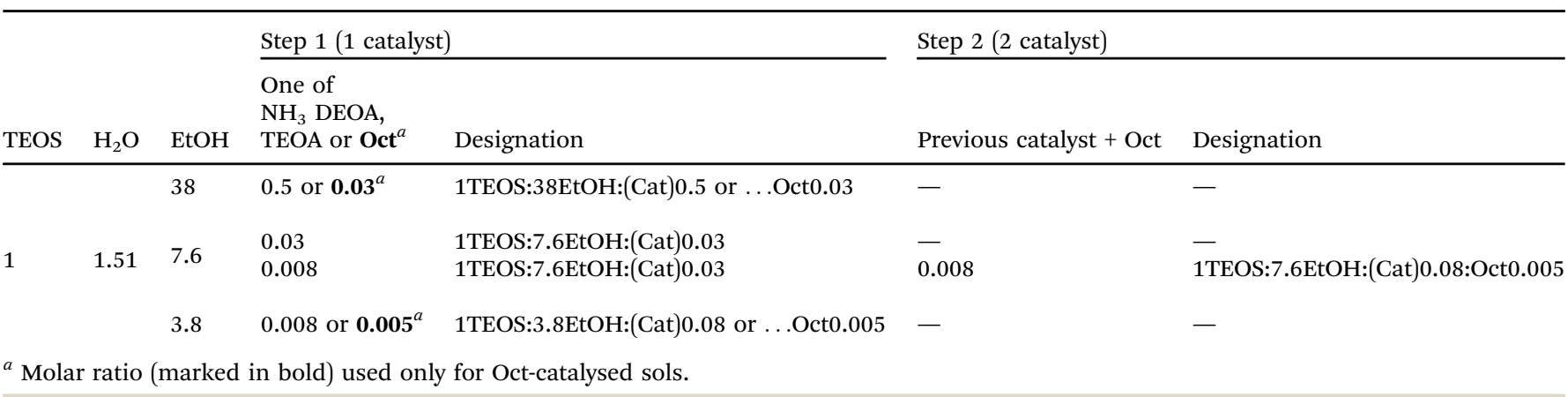

("Cat" being: $\mathrm{NH}_{3}$, Oct, DEOA, and TEOA). The solvent and catalyst ratios varied, but the TEOS to water molar ratio was kept constant at 1 TEOS $: 1.51 \mathrm{H}_{2} \mathrm{O}$ - following the same procedure. $^{27}$ The molar ratios of the mixture are summarised in Table 1. The resulting materials are denoted by their EtOH: (Cat) molar ratio, since these represent the main variables in relation to TEOS, as for instance 1 TEOS : $38 \mathrm{EtOH}: \mathrm{NH}_{3} 0.008$.

The apparent $\mathrm{pH}\left(\mathrm{pH}^{\prime}\right)$ values of the sols were measured using a pH meter (ProfiLine pH 3110) before and after addition of the catalysts, its role being crucial in the sol-gel route and in the mechanisms of gel formation.

Preliminary trials (controlling $\mathrm{pH}^{\prime}$, final sols behaviour, gelling and drying times) allowed the selection of the aforementioned variations in terms of TEOS:(Cat) molar ratios. The choice for the addition of octylamine in a second step, in some cases, took into consideration the surfactant-templating effect, ${ }^{19}$ which would prevent precipitation. Octylamine has been referred to as a more efficient catalyst compared, for instance, to ammonia, reducing the gelling time by few orders of magnitude. This can render a positive feature when trying to avoid coagulation, which can occur during the process. ${ }^{28}$

\subsection{Drying environments and sol characterisation}

To study and characterise the xerogel, to understand better the sol-gel mechanism and the impact of the carbonate environment in each system, three procedures were adopted according to previous studies: ${ }^{5,20}$

(a) $15.0 \mathrm{~mL}$ of sols were placed inside cylindrical flasks (3.5 cm diameter) with pinholes (needle-size) in the cap (hereafter, xerogels);

(b) $10 \mathrm{~mL}$ of sols were mixed with $10 \mathrm{~g}$ of the $\mathrm{CaCO}_{3}$ powder and placed inside cylindrical flasks (hereafter, calcite blends);

(c) sols were applied to limestone samples $(30 \times 30 \times$ $30 \mathrm{~mm}^{3}$ ) by capillary suction for 3 hours.

All samples were kept in a chamber at $20 \pm 5{ }^{\circ} \mathrm{C}$ and $60 \pm$ $10 \%$ relative humidity $(\mathrm{RH})$.

All parameters evaluated for each one of the resulting materials (xerogel, calcite blends and treated stone samples) are summarised in Table 2 . An early screening process, which included the observation of the homogeneity, gelling time and cracking tendency of the xerogels, was determined for assessing sol viability as consolidating materials, allowing to exclude non-viable sols. The excluding criteria are highlighted in Table 2, according to the procedure reported by Sena da Fonseca et $a .^{29}$ The stability of xerogels, calcite blends and treated stone samples was assessed over time using weight measurements, until the weight changes were $<0.01 \mathrm{~g}$ over 7 days.

The preliminary cohesion ability of the sol was assessed through the determination of the cohesion class of calcite blends - monoliths manually broken after stabilisation and their relative resistance was established (Table 2). Despite dependence on the operator, this expedite assessment of the sols being more suitable for consolidation is a useful and simple test. ${ }^{5,13,20}$

Shore A hardness measurements were used to characterise the calcite blend hardness. A Shore A durometer type (LCD Display Meter 0-100 HD), with a standardised presser foot (hardened steel rod (SR) 1.1-1.4 mm diameter, with a truncated $35^{\circ}$ cone, and a $0.79 \mathrm{~mm}$ diameter truncated tip) was used to measure the depth of indentation in the material to a given force of $8.1 \mathrm{~N}$. The resulting value registered (between 0 and $100 \mathrm{HD}$ ) corresponds to the resistance of the material to indentation.

\subsection{Performance on limestone samples}

Key parameters influencing the performance of the treatment were firstly assessed during the application of the sols onto Ançã stone samples, specifically, the capillary fringe evolution and absorbed sol (variation of weight after $3 \mathrm{~h}$ of capillary suction, see Table 2). These are indicative of the penetration ability within a porous system of the sols. The potential consolidating efficacy of selected sols was evaluated by the increments caused on the characteristic hardness and drilling resistance of Ançã stone. The colour variation of the limestone after the sol application was investigated as being one of the most evident and critical compatibility indicators.

Shore D hardness measurements were used to characterise the hardness of the treated limestone surfaces where a treatment with the obtained sols was applied. A Shore D durometer (LCD Display Meter 0-100 HD) with a standardised indenting foot (hardened steel rod with a $30^{\circ}$ cone, and a $0.1 \mathrm{~mm}$ radius tip) was used to measure the depth of indentation in the material to a given force of $44.5 \mathrm{~N}$. When the foot penetrated 
Table 2 Summary of the parameters evaluated for characterisation and interpretation of the prepared sols' potential to consolidate cabonate-stone

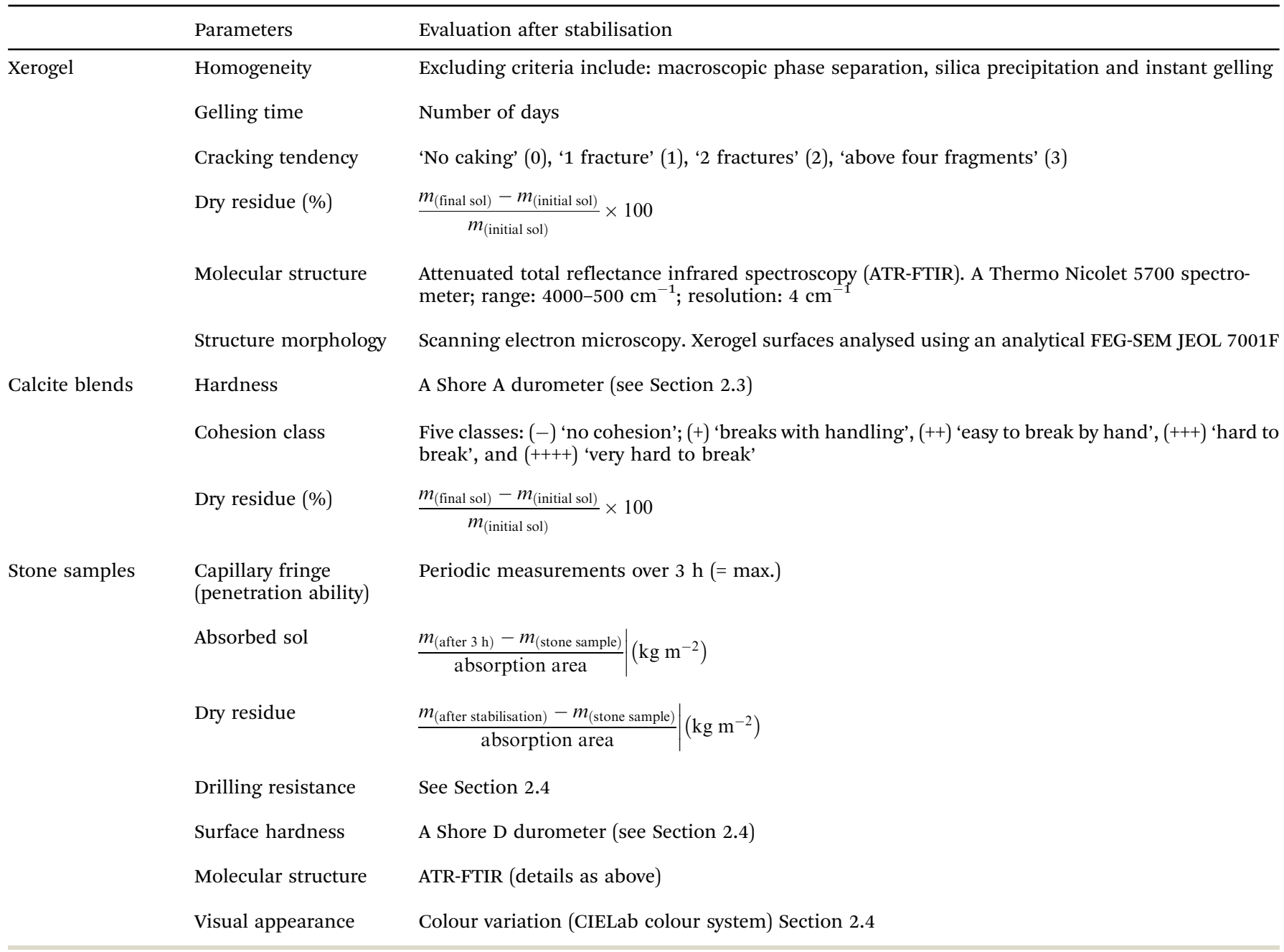

the surface of the material, the resistance of the material to indentation was registered (between 0 and $100 \mathrm{HD}$ ). A minimal sampling of six measurements per stone surface was carried out.

Drilling resistance was measured across the sample depth. To minimise any eventual disturbance of the data acquired caused by the packing of stone powder, a guide hole with $3 \mathrm{~mm}$ of diameter was performed across $30 \mathrm{~mm}$ of the samples. The test was made by using a $5 \mathrm{~mm}$ diameter diamond drill bit to drill over the guide hole and the resultant powder was vacuumed through the opposite side of the guide hole during the drilling. All drilling tests (minimal sampling of three holes per stone sample) were performed at a

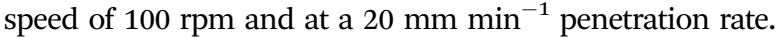

Total colour variation $\left(\Delta E^{*}\right)$ - a compatibility indicator was assessed with a colorimeter (KangGuang WSD-3A). The colourimetric coordinates $\left(L^{*}, a^{*}\right.$, and $\left.b^{*}\right)$ were registered (minimal of three measurements per surface) before and after treatment by capillary suction (e.g. $\Delta L^{*}=L_{\text {after }}^{*}-L_{\text {before }}^{*}$ ). The difference between the average values was calculated by $\Delta E^{*}$, as follows:

$$
\Delta E^{*}=\sqrt{\left(\Delta L^{*}\right)^{2}+\left(\Delta a^{*}\right)^{2}+\left(\Delta b^{*}\right)^{2}}
$$

\section{Results and discussion}

\subsection{Sols and xerogels}

Both, highly cracked and a small group of monolithic xerogels were obtained by drying inside cylindrical flasks with pinholes in the cap (Fig. 2). Since the gelling time is a determinant in terms of application of the consolidating material, some of the sols were excluded. The reason for this is that the reactions must occur within an optimal period of time, allowing the penetration into the stone pores, but at the same time avoiding excessive exposure to environmental parameters that can interfere with the sol-gel reactions, route and transition. ${ }^{20}$ Thus, based on the trials performed, there was immediate exclusion of some of the sols (marked with $\otimes$ in Fig. 2) due to one of the following reasons: instant gelling, macroscopic phase separation or silica precipitation, which are undesirable phenomena that invalidate the sols as potential stone consolidation products. ${ }^{29}$

Despite the fact that not all gels were completely colourless or without cracking, it was considered that this was not an excluding parameter. ${ }^{29}$ Relative importance should be given to this factor: being a different gelling environment compared to 


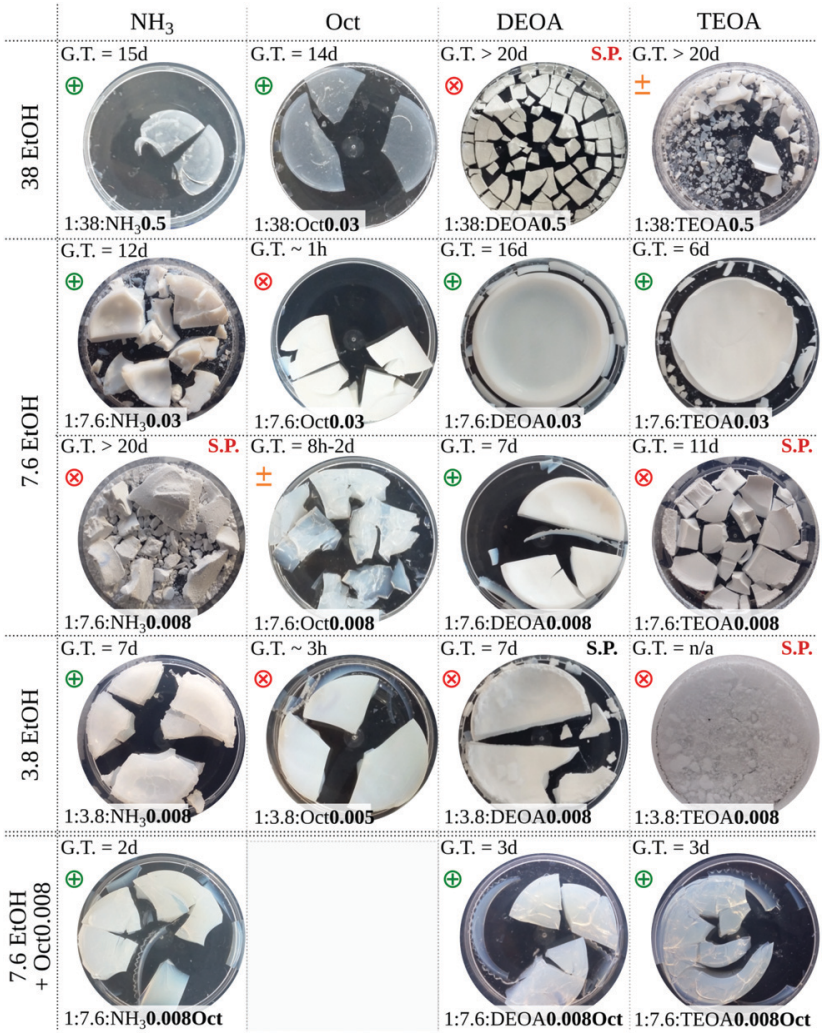

Fig. 2 Top views of the xerogels produced from the molar ratios presented in Table 1. Legends of the represented parameters: G.T. = gelling time, S.P. $=$ silica precipitation, $\oplus=$ adequate gelling time; $\pm=$ risk of too short $/$ too long gelling time; $\otimes$ = excluded sol due to non-compliance with one of the parameters listed in Table 2.

the stone pores, ${ }^{5}$ there may be a chance that potentially positive results can be obtained within the carbonate-medium.

3.1.1 Effect of different amines as basic catalysts. The effect of different amines and ammonia on the morphology of the silica structure/particles needs to be discussed considering the probable reaction mechanisms.

The $\mathrm{pH}^{\prime}$ values ${ }^{13,20}$ of the reaction system are useful for the understanding of the sol-gel process and silica formation; hence the resulting measurements are summarised in Table 3. The sols' basic properties are related not only with the $\mathrm{p} K_{\mathrm{a}}$ values of the amines, which vary in the order Oct (10.7) $>\mathrm{NH}_{3}$ (9.3) > DEOA (8.7) $>$ TEOA (7.7), ${ }^{30}$ but also with amine concentration, as seen in Table 3. The stronger the base and

Table $3 \mathrm{pH}^{\prime}$ of the sol registered upon the addition of each catalyst

\begin{tabular}{llllll}
\hline EtOH & (Cat) concentration & $\mathrm{NH}_{3}$ & Oct $^{a}$ & DEOA & TEOA \\
\hline 38 & $0.5 / 0.03^{a}$ & 10.84 & 10.76 & 10.42 & 8.99 \\
& & & & & \\
7.6 & 0.03 & 9.91 & 10.76 & 9.15 & 8.60 \\
& 0.008 & 9.50 & 10.37 & 9.35 & 8.14 \\
3.8 & $0.008 / 0.005^{a}$ & 9.56 & 10.14 & 9.64 & 8.23 \\
7.6 & $0.008+0.008$ Oct & 9.51 & - & 9.25 & 8.11
\end{tabular}

${ }^{a}$ For sols catalysed with Oct. the higher its concentration, the higher the concentration of $\mathrm{OH}^{-}$in the medium - thus increasing the rate of sol-gel reactions. Sols synthesised at higher pHs are expected to have more hydrolysed species, ${ }^{31}$ in this case corresponding to the highest molar ratio of each amine $(0.5 / 0.03)$ or the amine with the highest $\mathrm{p} K_{\mathrm{a}}$ value (Oct).

The silica network growth mechanisms at such pHs (above 7) are different from the ones below 7 , as in alkaline media most of the silica growing particles become appreciably charged and repel each other (most silanol groups are deprotonated), minimising collisions and, thus, condensation between distinct silica spheres. Furthermore, the higher solubility of silica causes particle growth through the Ostwald ripening processes, in which smaller and more soluble particles dissolve and supply high mobility monomers to larger particles through re-precipitation..$^{32}$ The resulting material is constituted by large silica particles, which sizes depend on the $\mathrm{pH}$ value. In extreme cases, these spherical particles may precipitate before gelation. ${ }^{33}$ The presence of salts or other molecules that can act as buffer ions modify the activity of the precursors and play important roles in the formation of gels or particulate structures by reducing, or neutralising, the charge repulsion. ${ }^{33}$

The catalysts used for the prepared sols are known to produce $\mathrm{OH}^{-}$ions and their conjugated acids in the form of $\mathrm{RH}_{(n+1)} \mathrm{N}^{+}{ }^{25}$ The latter are likely electrostatically attracted to the negatively charged deprotonated silanols, i.e. to the surface of the developing silica particles.

To obtain information about the structural bonding characteristics and potential evidence of sol modification by the action of each catalyst, ATR-FTIR analyses were conducted after the stabilisation period (minimum 2 months). Fig. 3 shows the spectra of several base-catalysed xerogels. In the region of $1300-700 \mathrm{~cm}^{-1}$, the Si bonds are evident, characteristic of a silica network structure. The $\mathrm{Si}-\mathrm{O}-\mathrm{Si}$ bonds have asymmetric stretching $(\nu($ as $))$ longitudinal and transversal optical modes (LO, TO, respectively), at about $1200-1000 \mathrm{~cm}^{-1}$, and symmetric stretching $(\nu(\mathrm{s}))$ mode at $c a .790 \mathrm{~cm}^{-1}$, and the $\mathrm{Si}_{-} \mathrm{O}^{-}$bonds have an in-plane stretching mode at $c a$. $940 \mathrm{~cm}^{-1} \cdot{ }^{20,24,34}$ The most visible bands are seen in Fig. 3(a), where the concentration of catalyst is higher (corresponding to the 1TEOS:38EtOH:(Cat)0.5 - or 0.03 for Oct - xerogel series). Besides a skeletal Si-O-Si peak and a broad $\nu_{(\mathrm{O}-\mathrm{H})}$ band centred at $3400 \mathrm{~cm}^{-1}$, two kinds of $\nu_{(\mathrm{C}-\mathrm{H})}$ peaks were observed. Visible bands are:

(i) the $\nu(\mathrm{as})_{(\mathrm{C}-\mathrm{H})}$ and $\nu(\mathrm{s})_{(\mathrm{C}-\mathrm{H})}$ fundamental vibrations, strong at $c a .2930-2850 \mathrm{~cm}^{-1}$ for DEOA, at $c a .2950 \mathrm{~cm}^{-1}$ for TEOA, ${ }^{35}$ and also about $2950-60 \mathrm{~cm}^{-1}$ for $n$-octylamine; $;^{36,37}$

(ii) the out of plane $\nu_{(\mathrm{C}-\mathrm{H})}$ around $1420-1360 \mathrm{~cm}^{-1} ;{ }^{35}$ and the $\nu_{(\mathrm{N}-\mathrm{C})}$ at $1450 \mathrm{~cm}^{-137}$ for DEOA and TEOA;

(iii) the $\nu_{(\mathrm{C}-\mathrm{C})}, \nu_{\mathrm{O}-\mathrm{C}}$ and $\nu_{(\mathrm{N}-\mathrm{C})}$ vibrations of the amines - Oct, DEOA and TEOA - in the region of $1000-800 \mathrm{~cm}^{-1}$ of the respective spectra; ${ }^{34}$

(iv) the absence of all these vibrations (item (i) to item (iii)) in the $\mathrm{NH}_{3}$ catalysed xerogel spectra.

(v) a small shift of the peak assigned to the $\nu(\text { as) })_{(\mathrm{Si}-\mathrm{O}-\mathrm{Si})}$ modes to lower wavenumbers also being noticed in the cases of 


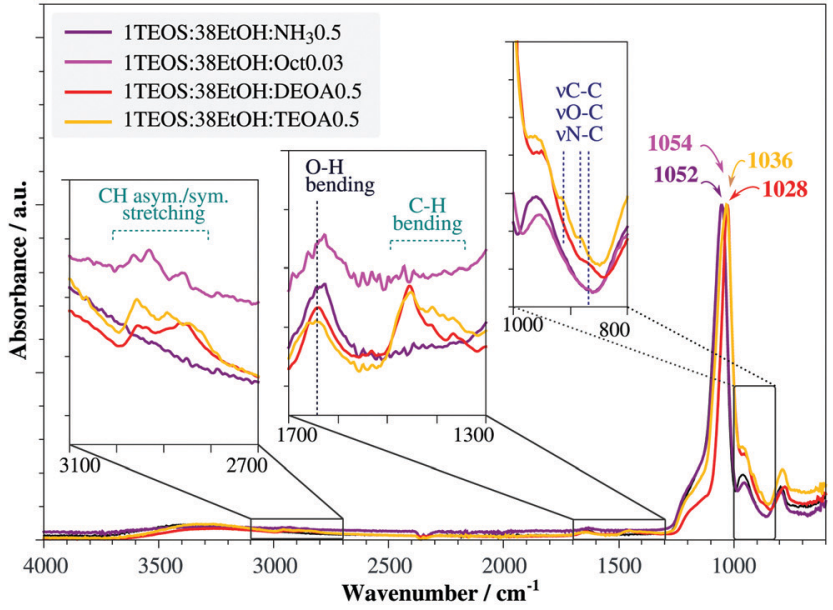

(a) Xerogels prepared with 1TEOS:38EtOH:(Cat)0.5 or 0.03 for Oct.

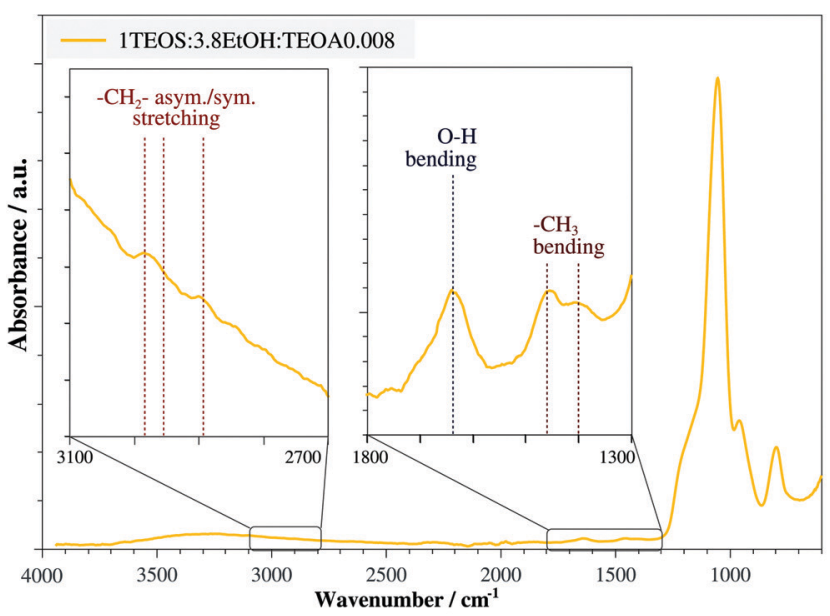

(c) Xerogel prepared with 1TEOS:3.8EtOH:TEOA0.008.

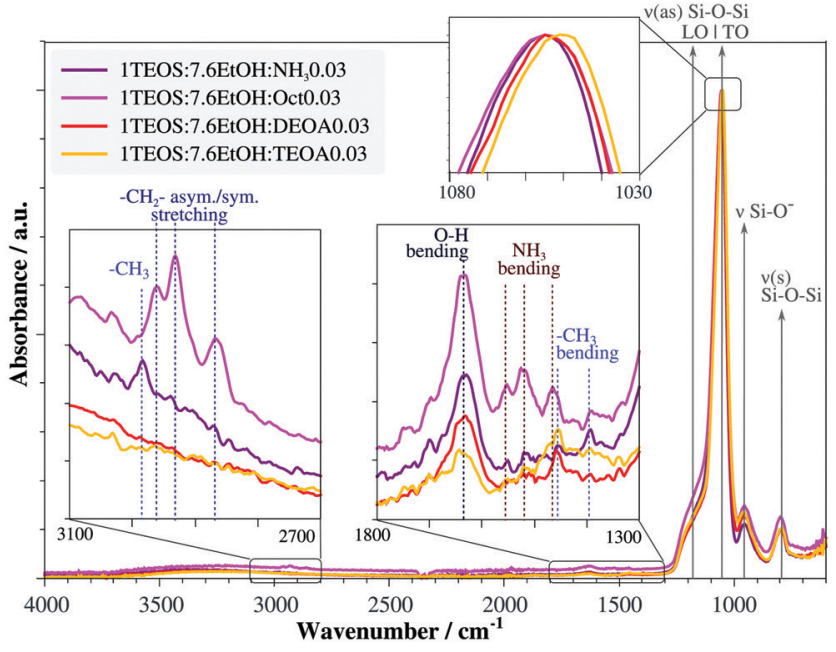

(b) Xerogels prepared with 1TEOS:7.6EtOH:(Cat)0.03.

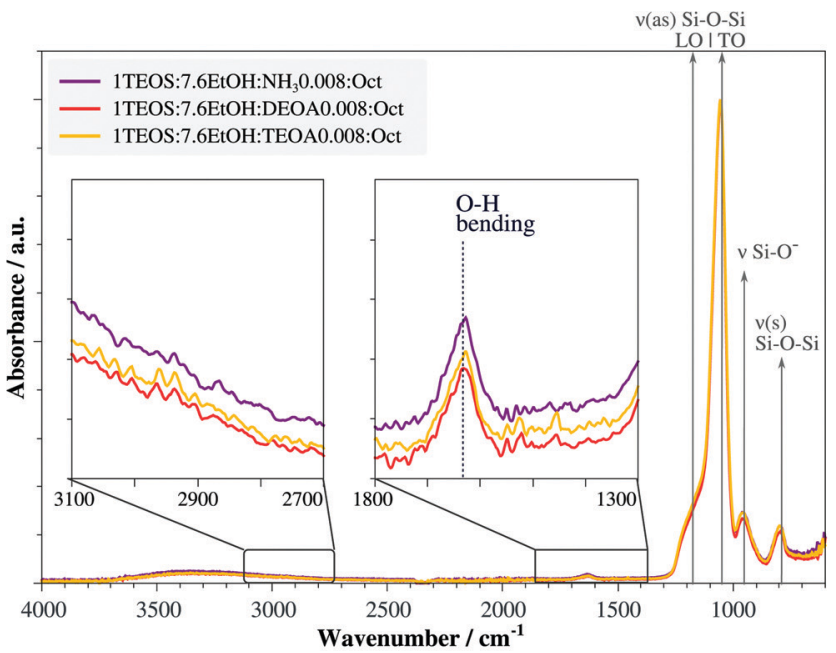

(d) Xerogels prepared with 7.6EtOH:1TEOS:(Cat)0.008 and addition of Oct0.008.

Fig. 3 Comparison of the FTIR spectra of several xerogels (stabilised over two months). Bands attributed according to ref. $34-37$.

DEOA and TEOA catalysis, suggesting a different structural environment.

In Fig. 3(b), where ATR-FTIR spectra of more concentrated formulations are compared (lower EtOH to TEOS and Cat ratio), the same $\mathrm{CH}$ vibrations are only evident in the $n$-octylamine catalysed xerogel. The spectra of the sols catalysed by DEOA and TEOA do not present clearly the $\nu_{(\mathrm{C}-\mathrm{H})}$ vibrations in the $3100-2700 \mathrm{~cm}^{-1}$ region, despite the fact that their existence is proved in the bending region presented between 1800 and $1300 \mathrm{~cm}^{-1}$. This may occur due to the overlapping with the broad $\nu_{(\mathrm{O}-\mathrm{H})}$ stretching vibration band centred at $3400 \mathrm{~cm}^{-1}$, which is more intense in these two cases likely due to the higher catalyst concentration, containing two or three $\mathrm{OH}$ groups (DEOA or TEOA, respectively). Additionally, in this particular series, a peak at $c a .2985 \mathrm{~cm}^{-1}$ is present in the $\mathrm{NH}_{3}$ catalysed sol, attributed to the $\nu_{\left(-\mathrm{CH}_{3}\right)}$ of ethoxy groups, probably due to their presence caused by incomplete hydrolysis of $\mathrm{TEOS},{ }^{38,39}$ which was induced in a higher concentration, when compared to the previous series of formulations (Fig. 3(a)).
The overall amount of $\mathrm{C}-\mathrm{H}$ bonds in xerogels with lower catalyst concentration (1TEOS:7.6EtOH:(Cat)0.008, Fig. 3(d), and 1TEOS:3.8EtOH:(Cat)0.008 series, Fig. 3(c)) is consequently lower and more difficult to detect. The absence of a clear signal at their characteristic IR bands in these series $\left(3100-2700 \mathrm{~cm}^{-1}\right.$ and $1800-1300 \mathrm{~cm}^{-1}$ regions) can be related to the ATR-FTIR equipment's detection limit. However, the example in Fig. 3(c) evidences low intensity bands present even for the lowest catalyst concentrations, hence being possible to infer the same for the other amines.

Therefore, these results reveal that each amine is integrated in the structure of the final xerogel, being present after the gel stabilisation period (above 2 months within the inert flasks). The slight shifts on the $\mathrm{Si}-\mathrm{O}-\mathrm{Si}$ bonds' asymmetric stretching bands also seem to confirm the differences in the several silica structures, likely resulting from the role of each catalyst.

The vibrations observed for $n$-octylamine might occur due to hydrogen bonding of the alkylamine to surface silanols, as 
reported in the literature ${ }^{19,28,40}$ and as follows:

$$
\begin{aligned}
& \equiv \mathrm{Si}-\mathrm{OH}+\mathrm{NH}_{2} \mathrm{C}_{8} \mathrm{H}_{17} \\
& \rightleftharpoons \equiv \mathrm{Si}-\ddot{\mathrm{O}}^{-} \cdots \mathrm{H}^{+} \ldots \mathrm{NH}_{2} \mathrm{C}_{8} \mathrm{H}_{17}
\end{aligned}
$$

The removal of this substance from the xerogel has been mentioned to occur by air drying in a period from 3 to 12 months, ${ }^{19}$ hence the presence of $n$-octylamine here was expected.

From the fact that the amine stretching and bending vibrations (e.g. $\nu_{(\mathrm{C}-C)}, \nu_{\mathrm{O}-C}$ and $\left.\nu_{(\mathrm{N}-C)}\right)$ are present in Fig. 3(a), it can be deduced that the integrity of the structural organic chains is maintained and that each alkanolamine (in this case, DEOA and TEOA) may bond with the silanols. Since during hydrolysis and condensation reactions the two alkanolamines may interact with the formed silanol groups mainly through hydrogen bonding, the possible bonding with the silanols may also occur with a similar interaction as in eqn (2), for DEOA (eqn (3)) and TEOA (eqn (4)), respectively:

$$
\begin{aligned}
& \equiv \mathrm{Si}-\mathrm{OH}+\mathrm{N} \mathrm{H}\left(\mathrm{CH}_{2} \mathrm{CH}_{2} \mathrm{OH}\right)_{2} \\
& \rightleftharpoons \equiv \mathrm{Si}-\ddot{\mathrm{O}}^{-} \cdots \mathrm{H}^{+} \cdots \mathrm{NH}\left(\mathrm{CH}_{2} \mathrm{CH}_{2} \mathrm{OH}\right)_{2} \\
& \equiv \mathrm{Si}-\mathrm{OH}+\ddot{\mathrm{N}}\left(\mathrm{CH}_{2} \mathrm{CH}_{2} \mathrm{OH}\right)_{3} \\
& \rightleftharpoons \equiv \mathrm{Si}-\ddot{\mathrm{O}}^{-} \cdots \mathrm{H}^{+} \ldots \mathrm{N}\left(\mathrm{CH}_{2} \mathrm{CH}_{2} \mathrm{OH}\right)_{3}
\end{aligned}
$$

Bands at around $2850 \mathrm{~cm}^{-1}$ corresponding to the $\nu_{(\mathrm{C}-\mathrm{H})}$ have been associated to H-bonding with silanol groups, ${ }^{41}$ thus the fact that these are present in the spectra of the sols catalysed by the alkanolamines may support this hypothesis. Bonding has been seen to occur, for instance, in the case of functionalisation of silicates with DEOA. ${ }^{42}$ Moreover, a shift in the $\nu(\mathrm{as})_{(\mathrm{Si}-\mathrm{O}-\mathrm{Si})}$ peak to lower wavenumbers can also be seen in the cases of DEOA and TEOA - Fig. 3(a). The latter could perhaps be correlated with a different structural environment and arrangement of silica bonds under the influence of small amounts of hydrogen-bonded catalyst in the sol structure. According to Nandy et al., ${ }^{25}$ the shift in the peak around $1100 \mathrm{~cm}^{-1}$ to lower waveumbers can be associated with the expansion of the $\mathrm{Si}-\mathrm{O}-\mathrm{Si}$ framework in the presence of the two alkanolamines.

Considering the presence of the amines in the final xerogels, SEM observations were performed to obtain information concerning the morphology of the resulting silica particles - Fig. 4 . The xerogel surfaces, in most cases were characterised by particles with diameters in the range of a few nanometres, contrary to results expected under acidic conditions. ${ }^{31}$ It becomes evident from the ATR-FTIR results (see Fig. 3), as well from the SEM results (see Fig. 4), that $\mathrm{NH}_{3}$, Oct, DEOA, and TEOA catalysts impact the microscopic textural and morphological characteristics of the sols.

When comparing the pHs of the sols (Table 3) with their morphology (Fig. 4), it is possible to conclude that no direct relation can be established. This confirms that the morphological modifications in these different catalyst systems are not solely governed by the $\mathrm{pH}$ of the sol-gel reaction. Contrarily, the macroscopic aspect of the xerogel (Fig. 2) can easily be correlated with its microscopic scale homogeneity, particle size and shape, and porosity (Fig. 4), resulting from the different catalyst action and different solvent ratios. The most homogeneous microstructures, with smaller and well-interconnected silica nano-particles (e.g. 1TEOS:38EtOH: $\mathrm{NH}_{3}$ )0.5, and 1TEOS:7.6EtOH:DEOA0.008:Oct) are related to 'glassy' and more transparent xerogels, whilst flocculated silica particles in a highly porous structure are correlated with silica precipitation in some cases (e.g. 1TEOS:7.6EtOH: $\mathrm{NH}_{3} 0.008$, 1TEOS:7.6EtOH:TEOA0.008, and 1TEOS:3.8EtOH:TEOA0.008).

3.1.2 Effect of amine chemical structure and concentration (same TEOS:EtOH). Under ammonia catalysis, TEOS is known to slowly condense and to form more spherical and uniform particles, which may become interconnected to form secondary particles ${ }^{31}$ - small-sized particles can be observed in Fig. 4, and their interconnection is inferred from Fig. 2. The $\mathrm{NH}_{4}{ }^{+}$cation formed during the reaction with water is electrostatically attracted to the surface of the growing silica particles, but its influence on their porosity is expected to be low due to the small size of the cation. Silica micro-spheres obtained with ammonia catalysis are reported to be non-porous, ${ }^{43}$ and the morphological features randomly form clusters as well as monodispersed $\mathrm{SiO}_{2} \cdot{ }^{27,30}$ The latter may depend on sol-gel parameters. In the presence of higher ammonia concentration (1TEOS:38EtOH: $\mathrm{NH}_{3} 0.5$, Fig. 4), there is a 3D ordering of these small particles, which can be explained by the increase in the ionic strength of the reaction medium with increasing ammonia concentration. ${ }^{44}$ In contrast, lower concentration of the catalyst in diluted sols resulted in silica precipitation, after a longer gelation period (1TEOS:7.6EtOH: $\mathrm{NH}_{3} 0.008$, Fig. 2 and 4).

Contrarily, when the primary amine, $n$-octylamine, is used as the catalyst, a microstructure with porous ultra-fine spherical silica nanoparticles ${ }^{45}$ that form a mesoporous material is expected. ${ }^{24,45}$ The produced xerogels show an overall tendency to form spherical uniform particles (Fig. 4), interlinked (inferred from Fig. 2), leading to a macroscopic more 'glassy' and translucid visual appearance (see Fig. 2). This amine acts as a templating agent and promotes the formation of silica particles with higher micro- to mesoporosity ${ }^{19,36,37,45}$ (coarser pore sizes $)^{40}$ due to its tendency to form reverse micelles that can be trapped inside the growing silica structure, ${ }^{26,37}$ reducing the capillary pressure. ${ }^{44,46}$ ATR-FTIR results presented in Fig. 3 suggest the incorporation of the catalyst molecules in the produced material. Octylamine can also act as a surfactant, by anchoring its cationic amine head at the negatively charged surface of the silica particles, whilst the non-polar tail prevents different particles to agglomerate. ${ }^{47}$ Likely, if particles grow around the catalyst molecule, it can be trapped into the silica structure, ${ }^{37}$ as suggested by ATR-FTIR (Fig. 3). The rapid formation of larger particles (compared to $\mathrm{NH}_{3}$ ) mediated by $n$-octylamine may occur due to its combined effect of a basic catalyst (high $\mathrm{p} K_{\mathrm{a}}$ ) and a surfactant (reducing the repulsion of particles charges), allowing it to increase their rate of chaining and aggregation, ${ }^{46}$ which is in accordance with the tendency to reduce the gelation time compared to other basic catalysts. ${ }^{28}$ 

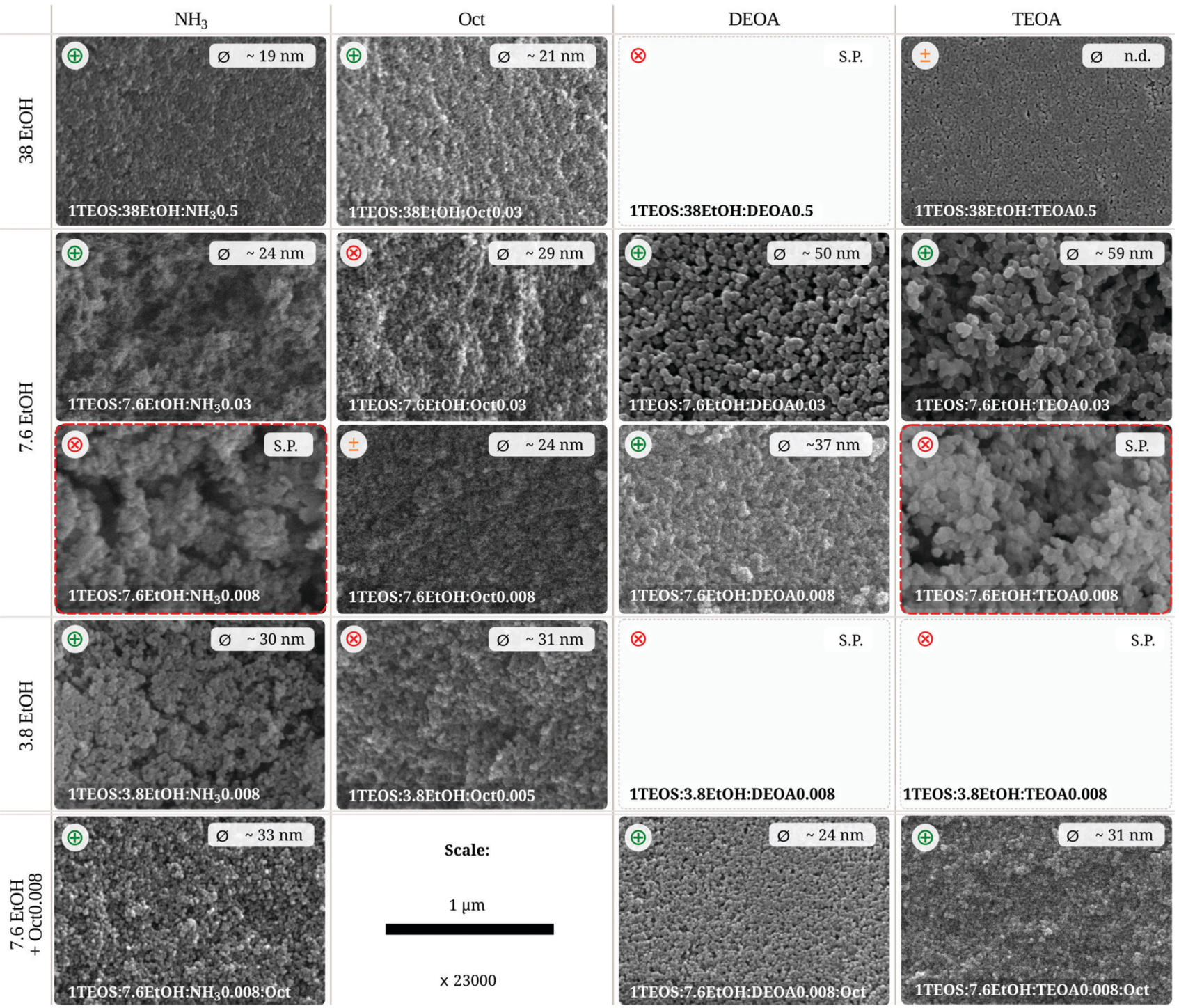

$\times 23000$

\section{TEOS:3.8EtOH:TEOA0.008}

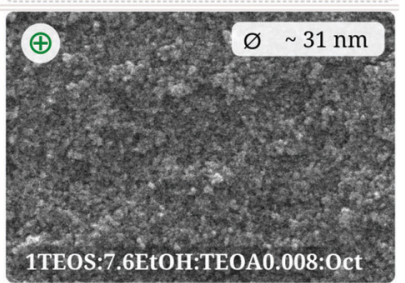

Fig. 4 SEM micrographs of the xerogels produced. Microstructures vary from well-connected silica to flocculated porous materials. On top of each image, the average particle diameter is indicated. Legends: S.P. = silica precipitation - micrographs also marked in red contour $-; \oplus=$ adequate gelling time; $\pm=$ risk of too short/too long gelling time; $\otimes=$ excluded sol.

Secondary and tertiary amines - in this case DEOA and TEOA - possess lower basicity and nucleophilic character. As in the case of $n$-octylamine, ionic interactions between the positively charged amine head and negatively charged silanols can increase the rate of the reactions of hydrolysis and condensation..$^{25}$ Nonetheless, in addition to the electrostatic attraction and anchoring on the negatively charged particle surfaces, DEOA and TEOA are hydroxyl-terminated (with 2 and $3 \mathrm{OH}$ groups, respectively), and can take part in further condensation reactions, either contributing to the growth of each particle or to the coalescence of several nuclei. Hence, when comparing the two 1TEOS:7.6EtOH sols catalysed with 0.03 and $0.008 \mathrm{DEOA} / \mathrm{TEOA}$, it is possible to observe that the increase in the catalyst content results in well connected networks composed of larger well-defined silica nano-spheres (Fig. 4). The entrapment of these amines is likely to occur (see Fig. 3(a)) and increased particle mesoporosity is also expected in the order TEOA > DEOA, according to previous reports. ${ }^{25}$ Due to the described effects, TEOA is also known as a dispersion agent and as a co-inhibitor of particle growth, ${ }^{48}$ since it is more sterically hindered (less nucleophilic character) compared, for instance, with $\mathrm{NH}_{3}$ and DEOA. ${ }^{25}$ The higher steric hindrance introduces a retarding effect on the hydrolysis and condensation reaction of TEOS, ${ }^{25,31}$ which would explain the resulting very small monodispersed and interlinked silica nano-structures observed in the case of 1TEOS:38EtOH:TEOA0.5 (Fig. 4).

The combined effect of $n$-octylamine as a basic catalyst and a surfactant clearly affects the reaction rate. Addition of $n$-octylamine in a second step, during the condensation of formulations produced using other catalysts - in the proportion 1TEOS:7.6EtOH:(Cat)0.008:Oct0.008 - likely promotes chaining of the already formed particles (under $\mathrm{NH}_{3}$, DEOA and TEOA catalysis). These particles are better interconnected compared 
to the case of the use of individual catalyst (Cat); the gelation time was reduced and silica precipitation was prevented.

3.1.3 Effect of TEOS concentration (same TEOS:Cat). In ammonia catalysed TEOS reactions, the sols with the ratios 1TEOS : 3.8EtOH : $\mathrm{NH}_{3} 0.008$ and 1TEOS : 7.6EtOH : $\mathrm{NH}_{3} 0.008$ present the same TEOS : $\mathrm{NH}_{3}$ ratio (fixed at $1: 0.008$ ). Therefore, by comparing them, the effect of the solvent-to-TEOS concentration in the final network can be observed. In the presence of lower concentration of ethanol, the proportionally higher concentration of TEOS results in smaller silica particles (Fig. 4), interlinked (inferred from Fig. 2) to form a more ordered 3D network. The influence of the higher concentration of reactive species in the medium could be the reason for agglomeration of particles within a lower gelation period. Contrarily, the 1TEOS:7.6EtOH: $\mathrm{NH}_{3} 0.008$ sol has lower concentration of reactive species. Additionally, this sol presented the lowest $\mathrm{pH}^{\prime}$ (Table 3), which is expected to drop to even lower values after the early stages of the reaction, due to the formation of silicic acid (\#SiOH) as the TEOS hydrolysis proceeds. ${ }^{49,50} \mathrm{~A} \mathrm{pH}$ decrease to neutral values may affect the degree of ordering in the $3 \mathrm{D}$ structure, ${ }^{49}$ which in turn may be responsible for the colloidal instability of this sol.

The $n$-octylamine-catalysed sol with higher solvent content (1TEOS:38EtOH:Oct0.03) rendered smaller particles within longer gelation periods compared to the sols with more precursor species. The fixed TEOS : Oct ratio - in 1TEOS : 7.6EtOH : Oct0.03 and 1TEOS : 38EtOH : Oct0.03 - allows comparison of the two sols. The influence of the protic solvent must be considered here too: by retarding the base-catalysed condensation, ${ }^{31}$ in this particular case, it contributes to decrease the size of the nanoparticles, and to slow down the condensation of the silica network.

Overall, the aggregation of particles seems to be slower in sols catalysed by DEOA and TEOA compared to that of $n$-octylamine, perhaps due to the higher amount of mutually repulsive charges in the sol $^{31}$ being not compensated by the effect of the $\mathrm{RH}_{(n+1)} \mathrm{N}+$ species in some cases. This likely occurs due to the lower basicity and nucleophilicity of these alkanolamines. The slower sol-gel reactions seem to affect the homogeneity of silica particles due to possibly the Ostwald ripening phenomenon - influencing the growth of primary particles, rather than the occurrence of chaining or aggregation. Hence, the 1TEOS:3.8EtOH:DEOA0.008 sol resulted in silica precipitation, contrary to the 1TEOS:7.6EtOH:DEOA0.008 sol where the same TEOS:DEOA ratio was used. When comparing 1TEOS:38EtOH:TEOA0.5 and 1TEOS:7.6EtOH:TEOA0.03 and/or 1TEOS:7.6EtOH:TEOA0.008 sol morphology, the same phenomenon seems to occur, since in the latter cases it seems to contribute to the formation of larger particles (Fig. 4). With the increase in TEOS: EtOH ratio, this growth can perhaps be explained by the formation of heterogeneous sized particles with an increase in the amount of the precursor (TEOS), possibly due to the formation of new nuclei amongst the already existing ones as previously proposed. ${ }^{44}$ In the case of 1TEOS:38EtOH:TEOA0.5, the large amount of the catalyst seems to prevent precipitation, which can be attributed to the higher steric hindrance. ${ }^{25,31}$ The formation of monodispersed interlinked silica spheres (Fig. 2 and 4) probably occurs because of the previously mentioned influence of the three - $\mathrm{OH}$ groups for the coalescence of several nuclei.

The increase in TEOS : EtOH molar ratio seems to contribute to the lower gelation periods in the cases of $\mathrm{NH}_{3}$ - and Octcatalysed sols, whilst in DEOA and TEOA catalysed sols it apparently contributes to a lower self-aggregation of larger and heterogeneous-sized particles, sometimes resulting in silica precipitation (see Fig. 2 and 4).

\subsection{Influence of the carbonate medium: calcite powder blends}

As previously presented, the microstructure of xerogels was found to be mainly dependent on the basic catalyst - i.e. on the role of each type of amine on the silica particles/network formation - and it varied from well-connected silica nanospheres to flocculated porous materials. The xerogels marked with $\oplus$ and \pm in Fig. 2 were selected for further blending with calcite powder, where the influence of the carbonate environment could be better assessed. It is known that the alkaline environment of the calcite blends influences and may inhibit condensation reactions (occurring in acid catalysed sols), resulting in a decrease of the extension of the final network of the xerogel. ${ }^{20}$ The choice for a pre-condesation alkaline environment was intended to reduce the influence of the carbonate medium, and it resulted - as shown in Fig. 5 - in some of the sols not forming solid materials with the ability to aggregate calcite powder. Even if some of the base-catalysed sols developed unfavourable microstructures for an efficient consolidation, there were cohesive monoliths of calcite powder within the selection of sols applied (see $\oplus$ and \pm marked sols in Fig. 5).

The expected role of the alkaline nature of calcite $(\mathrm{pH} \approx 9.5-9.7)^{3}$ would be in this case less pronounced for the sol-gel processes, since most of the pre-condensation conditions were at similar or at higher $\mathrm{pH}^{\prime}$ values, as shown in Table 3 . Hence, $\mathrm{pH}$ influence seems neither to induce significant changes in the relative rates of sol-gel reactions - as would be in the case of an acid catalysis, nor to change the condensation paths - as expected in acidic pre-condensation conditions. ${ }^{13}$ Therefore, the effect of the $\mathrm{Ca}^{2+}$ ions on screening repulsive charges may be the most determining factor, influencing both condensation and coagulation processes. In this case, it can be suggested that the coagulation of particles, rather than the gelation of the sol may occur, and that potentially there is formation of isolated and discontinuous aggregates of silica, rather than an interconnected silica nanoparticle covering film. Perhaps, this could be related to an excess of precursor evaporation, ${ }^{3}$ and lower cohesion when applied to calcite powders. This subject is still not fully understood, and more research seems necessary.

Calcite blends containing TEOA as the catalyst seem to produce monoliths with lower cohesion strength - e.g. Shore A hardness measurements were not possible due to the very low cohesion, except when Oct was added - whilst the three other catalysts seem to have produced at least one monolith with a higher cohesion degree. The addition of $n$-octylamine in 


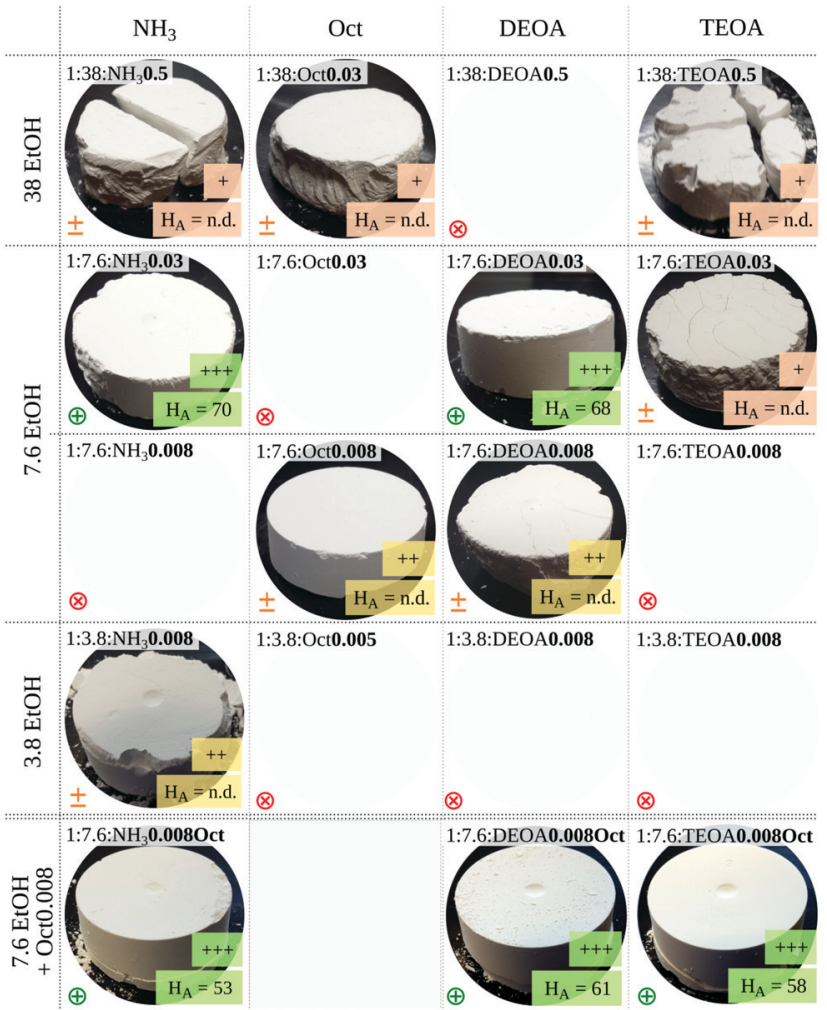

Fig. 5 Side views of the calcite blends produced with the sols previously presented. Both the cohesion class (see Table 2) and the Shore A hardness $\left(H_{A}\right)$ measurements are presented at the bottom right of each window. Symbols in the bottom left represent: $\oplus=$ high cohesion; $\pm=$ intermediate to low cohesion; $\otimes=$ no cohesion, excluded sol.

a second step also seems to have contributed to an increase in the cohesion degree of the respective monoliths (last row in Fig. 5).

\subsection{Consolidation potential: limestone samples}

The influence of the base catalysis with ammonia and amines on the efficacy of TEOS-based sols for the consolidation of carbonate stones was investigated through the treatment of sound limestone samples with the selection of most promising sols. The capillary fringe was used as an indicator of the depth of penetration of the selected sols, and the applied sols reached the top of the $3 \mathrm{~cm}$ porous stone samples tested within the limit of the application period $(3 \mathrm{~h})$.

The spectra of stones treated with the formulations (superficial layer) with higher amounts of catalysts are presented in Fig. 6. These are compared with that of the untreated limestone (Fig. 6(a)) and the subtracted resulting absorbance values are shown (Fig. 6(b)). Calcite $\left(\mathrm{CaCO}_{3}\right)$, being the major constituent of the stone, presents characteristic IR absorption bands around 1400, 870 and $712 \mathrm{~cm}^{-1} \cdot{ }^{20}$ Besides, small amounts of quartz $\left(\mathrm{SiO}_{2}\right)$ can also be present in calcite samples. Calcite and quartz can interfere with the interpretation of these spectra, since calcite unfortunately absorbs infrared radiation in the same region as the $\mathrm{C}-\mathrm{H}$ bond, ${ }^{1}$ whilst quartz's infrared absorption overlaps with the silane bond absorption (both $\mathrm{SiO}_{2}$ structures). Nonetheless, it is possible to observe through the

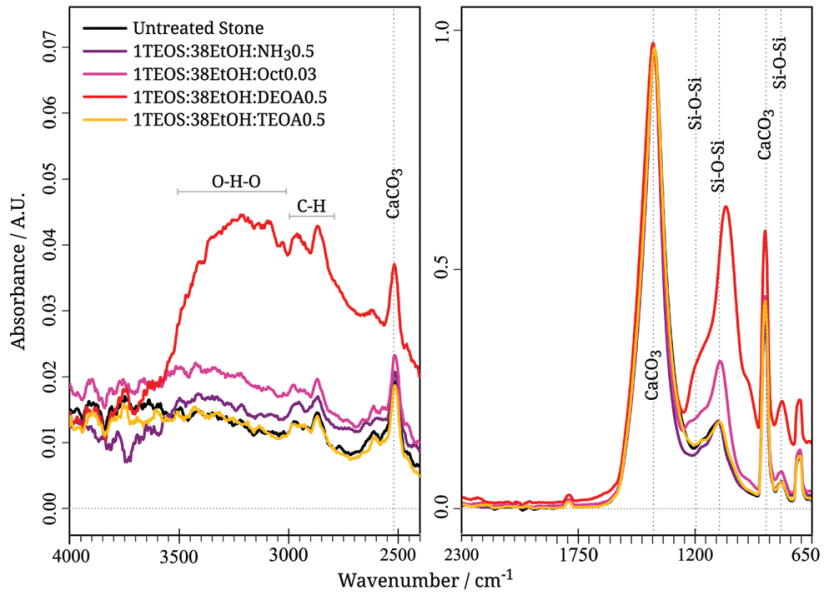

(a) Full spectra, normalised and compared with the untreated stone surface spectrum.

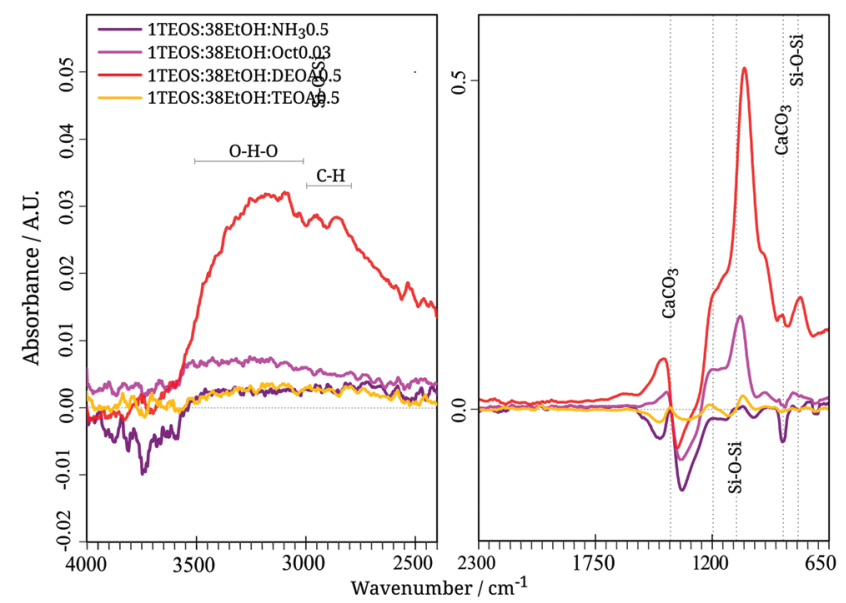

(b) Subtraction of the FTIR spectrum of the untreated stone surface applied to the treated stone FTIR spectra.

Fig. 6 FTIR spectra of the surfaces of the stone samples after treatment with the 38 (Cat) 0.5 xerogels.

subtraction of the spectra, that there is an increase in the absorbance in the $\mathrm{Si}-\mathrm{O}-\mathrm{Si}$ region when the stone is treated with several sols (e.g. DEOA and Oct treated surfaces), corresponding to the presence of the absorbed silane material. It is worth mentioning, however, that the band intensities related to the sols used for stone treatment may be dependent on their different concentrations (i.e. different dry residues), thus only their presence or absence is considered here.

The obtained data do not allow us to draw any conclusion on the influence of the $\mathrm{NH}$ groups of the ammonia and amine catalyst on the increase of the affinity to the carbonate stone surface. As previously observed by other authors, ${ }^{5}$ the $\mathrm{NH}$ groups influence the final network structure, and due to their small fraction there is no evidence (in this study or elsewhere ${ }^{5}$ ) of their interaction with the stone surface.

Concerning the consolidating potential of the synthesised sols, these need to fulfil other requirements to achieve proper stone consolidation. The gelling and drying times are very relevant features for a sol to be used as a stone consolidant; 
hence these parameters were considered in the initial screening process for selection of sols with consolidation potential. The solid materials (often designated dry residue) left within the stone pores by stone consolidants such as alkoxysilanes can contribute to the enhancement of the stone's integrity by two different mechanisms: (i) filling the interstices between the loosened grains or (ii) by forming adhesive bridges between adjacent grains and this way maintaining them together., ${ }^{3,51}$ Additionally, a relevant requirement is the depth of penetration of the product, which needs to be compatible with the degraded thickness of the stone..$^{2,20,29,52}$ Independently of the consolidation mechanism, the enhancement of stone integrity is sought in order to minimise or avoid the fast rate of material loss. ${ }^{3}$ Hence, if the sols provide a resistance increase in depth, they have potential to be used for the consolidation purpose. Several authors mention minimal penetration depths of $15-20 \mathrm{~mm}$ (e.g. Sena da Fonseca et al., ${ }^{3}$ and Rodrigues and Grossi ${ }^{53}$ ).

To assess the depth up to which there was an effective stone strength gain, the drilling resistance test renders better and more accurate results than the capillary fringe (Fig. 7). The average resistance of Ançã stone - used as a reference since it is very homogeneous - is presented for comparison and revealed to be around $4.6 \mathrm{~N}$. The average profiles presented for the sols show that not all tested sols promoted the increase of the stone resistance in depth (see Fig. 7(a)).

In the case of ammonia catalysis, the sols 1TEOS:38EtOH: $\mathrm{NH}_{3}$ 0.5, 1TEOS:7.6EtOH: $\mathrm{NH}_{3} 0.03$ and 1TEOS:3.8EtOH: $\mathrm{NH}_{3} 0.008$ were responsible for a significant increase in the resistance, whilst only a superficial increase (less than $1 \mathrm{~mm}$ thick) can be seen when $n$-octylamine is added in a second step reaction (1TEOS:7.6EtOH: $\left.\mathrm{NH}_{3} 0.008: O c t\right)$. Only one $n$-octylamine catalysed sol was responsible for a slight increase in the average resistance to drilling; (1TEOS:7.6EtOH: $\mathrm{NH}_{3} 0.008$ ) having low potential to act as a consolidating material. Moreover, DEOA was clearly less responsible for sols providing a strength gain in depth, due to the similarity observed between the resistance profiles of the treated and the untreated stone. A slight gain could be observed up to around $15 \mathrm{~mm}$ deep within the surface when $n$-octylamine was added in a second step (1TEOS:7.6EtOH:DEOA0.008:Oct). Finally, TEOA catalysed sols are the most surprising, given the low cohesion of the monoliths produced (see Fig. 5),
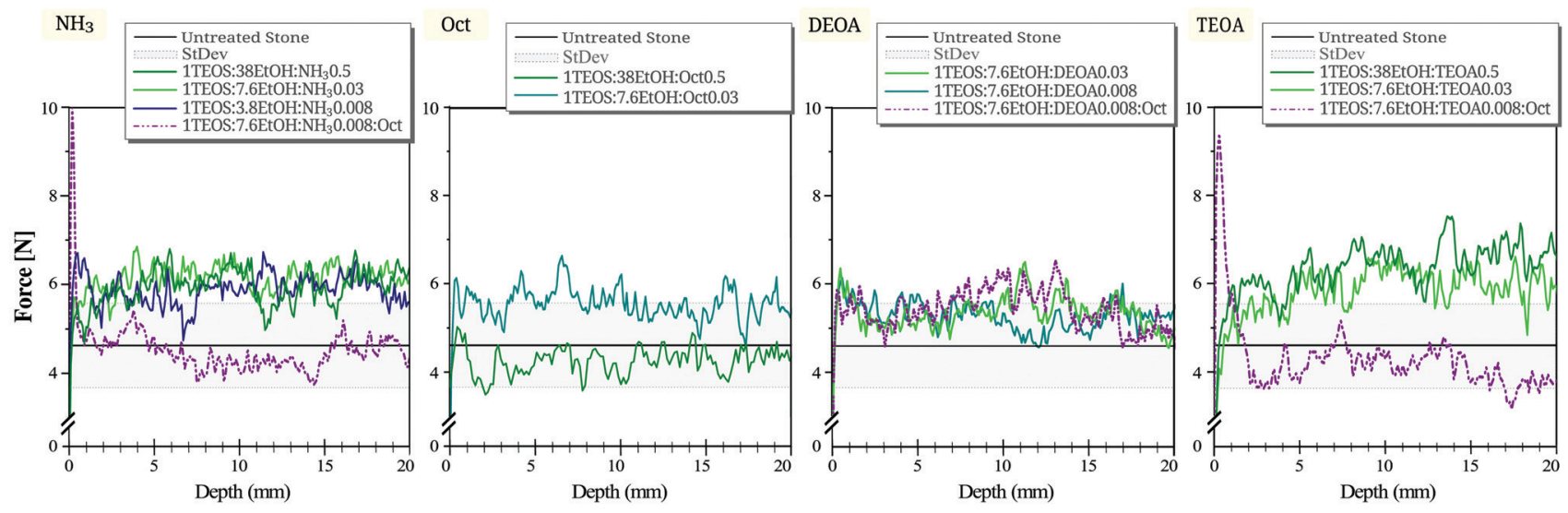

(a) Drilling resistance profiles of stones treated with the most promising sols - separated by catalyst. All yy axis present the same scale and units.
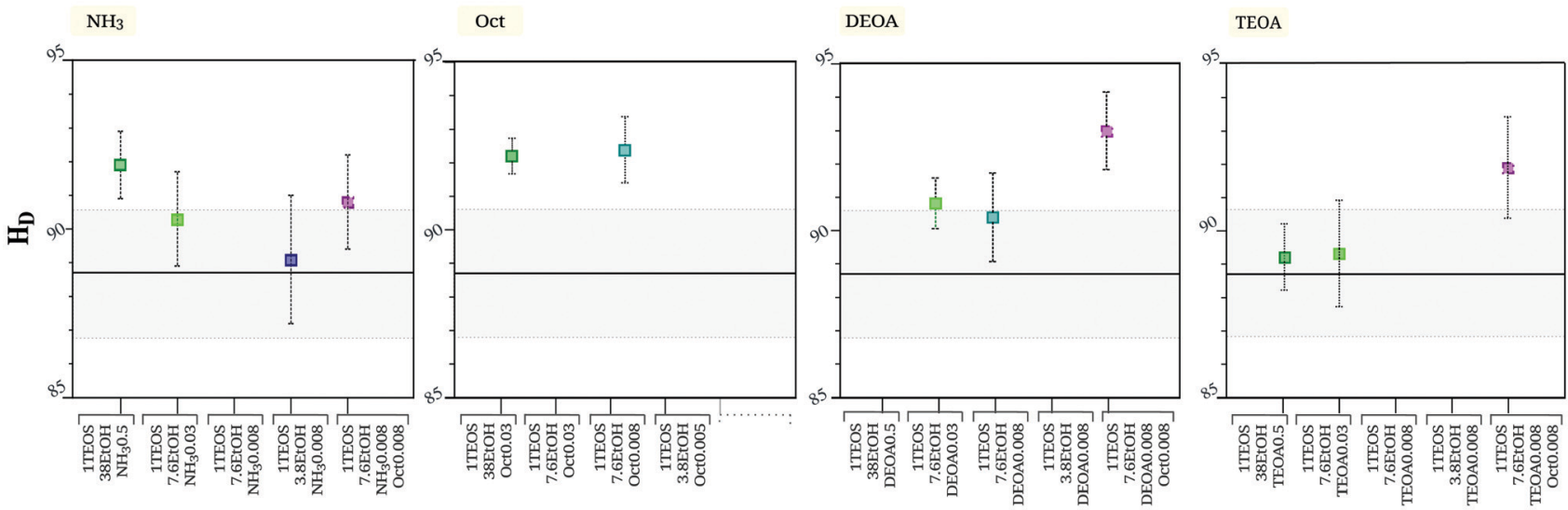

(b) Shore D Hardness measurements on the surface of stones treated with the most promising sols - separated by catalyst. All yy axis present the same scale and units, and legend of fig. 7(a) applies.

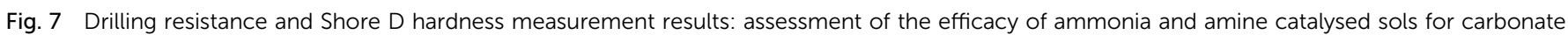
stone consolidation. 
opposed to a significant gain of strength in the cases of 1TEOS:38EtOH:TEOA0.5 and 1TEOS:7.6EtOH:TEOA0.03. Once again, the case where $n$-octylamine was added in a second step (1TEOS:7.6EtOH:TEOA0.008:Oct) was the least promising as a consolidating material, since a $2 \mathrm{~mm}$ 'crust' is followed by no increase in resistance to drilling in depth compared to the reference material.

Fig. 7 also shows plots of the surfaces' Shore D hardness comparing the treated samples with the untreated stone surface (Fig. 7(b)). There seems to exist a correlation between an increase of Shore D hardness of surfaces treated with the TEOA-catalysed sols (see Fig. 7(b) TEOA plot), and the increase of the drilling resistance of these surfaces in the first millimetre depth (see Fig. 7(a) TEOA plot). In some other cases, the increase of the stone surface's $H_{D}$ cannot be visibly related with a higher increase of the drilling resistance in the first millimetre depth. A reverse migration of the sol towards the surface may occur, resulting in the increase in the surface hardness, which in some cases was not identified in the drilling profiles possibly due to its occurrence in a very small thickness.

The highest drilling resistance increments were observed for more than one TEOS : EtOH ratio under $\mathrm{NH}_{3}$ and TEOA catalysis (around $30 \%$ and $40 \%$, respectively, in relation to that of the untreated stone). Interestingly, the higher increments are associated with the smaller silica particles (up to $\sim 30 \mathrm{~nm}$ diameter), and are well interconnected, which may be inferred from the observation of the xerogels (see Fig. 4) - the only exception being the 1TEOS:7.6EtOH:TEOA0.03 sol (larger particles, $\sim 59 \mathrm{~nm})$. Note that these morphologies might not be exactly the same when condensation keeps going inside the stone pores, as already discussed. However, and independent of the possible sol-gel path differences, it may be possible that the ability of $\mathrm{NH}_{3}$ as a catalyst, and of TEOA on limiting the growth and promoting aggregation of silicate species ${ }^{30}$ can somehow contribute to the positive results observed upon the stone specimens' treatment. An almost complete fill of intergranular space is not expected to be the most likely mechanism in place in the current scenario, but perhaps a covering layer and bonding of grains at contact points could occur ${ }^{3,51}$ (see for instance the final dry-residue values presented ahead in Table 5). This could be somewhat favoured in the case of $\mathrm{NH}_{3}{ }^{-}$and TEOA-catalysed sols within stone pores, as opposed to those of Oct and DEOA. If the resulting solid material from $\mathrm{NH}_{3}{ }^{-}$and TEOA-catalysed sols would follow this intermediate mechanism (thin film and bonds at contact points) - an interlinking tendency is observed in some of the corresponding xerogels (Fig. 4) - this could explain some differences of strength gain upon stone treatment with the four kinds of catalysts. Further studies are necessary to definitely conclude if the aforementioned factors affect and/or may be responsible for the formation of silica structures within stone pores that increase stone cohesion, rendering better results when compared with those of two other catalysts studied.

Concerning the increase in stone drilling resistance, authors also have established limits for a maximum gain, due to the potential risks for the substrate material and the formation of
Table $4 E^{*}$ (total colour variation) values of the treated stone surfaces by comparison with those of the untreated surfaces. Values equal to or above 5 (risk of incompatibility) are underlined

\begin{tabular}{|c|c|c|c|c|c|}
\hline EtOH & Cat conc. $/ \Delta E^{*}$ & $\mathrm{NH}_{3}$ & Oct $^{a}$ & DEOA & TEOA \\
\hline 38 & 0.5 & 2.7 & 3.1 & - & 1.8 \\
\hline \multirow{2}{*}{7.6} & 0.03 & 3.4 & - & $\underline{8.9}$ & 1.1 \\
\hline & 0.008 & - & $\underline{5.9}$ & 3.2 & - \\
\hline 3.8 & 0.008 & 2.4 & - & - & - \\
\hline 7.6 & $0.008+0.008 \mathrm{Oct}$ & $\underline{8.7}$ & - & $\underline{5.0}$ & $\underline{5.6}$ \\
\hline
\end{tabular}

interfaces between the consolidated and the underlying stone. These values vary from $25 \%$ to $300 \%$ relative to the untreated stone resistance. ${ }^{29,53}$ The values obtained here (see Table 5 ahead) are closer to a more conservative proposal - therefore appropriate for mild consolidation procedures.

The samples treated with the base-catalysed selected sols were submitted to colorimetric characterisation for the evaluation of the induced colour alterations (Table 4). According to the total colour variation values $\left(\Delta E^{*}\right)$, the samples revealed minor colour changes in general. Total colour variation above 5 can be considered a risk due to the fact that it can be perceptible to the naked eye. ${ }^{53}$ The small variations encountered in the treated surfaces are mainly due to an increase in the $b^{*}$ coordinate, which means an overall gain in the yellow tone. However, most changes are almost unnoticeable under macroscopic inspection; at the same time the highest $\Delta E^{*}$ values corresponded to the sols with the lower/least uniform strength gains from the drilling resistance results (Fig. 7), and therefore their potential was discarded.

Considering these and other relevant parameters, the improved sols for the consolidation of carbonate materials considered consist of the sols summarised in Table 5.

When evaluating the potential efficacy of newly developed consolidating products for decayed soft porous limestones that can be considered as one of the most challenging substrates - it has been defined that the (a) 'effectiveness', (b) 'harmfulness' and (c) 'durability' of the treatments are key factors in their potential for practical applications. ${ }^{53,54}$ When comparing the consolidation effects of the produced sols with others, it is necessary to consider not only (a) the strengthening effect of the consolidation, but also (b), the risk of incompatibility between the treatment and the stone, which can induce additional damages. Table 6 presents a summary of other consolidation products applied to the same type of limestone (Ançã stone), whenever possible through the evaluation of parameters for the same type of treatment - for comparison with the currently developed sols. It is well known that both the application procedures and the types of substrate influence the consolidation performance in such a way that even minor conditionins may have relevant implications in the final result. $^{2}$ Other types of products have been considered for this comparison besides alkoxysilane-based products (e.g. acrylic and epoxy resins, inorganic products and bio-consolidants). 
Table 5 Summary of the most relevant assessed parameters of the produced sols with improved properties for the consolidation of carbonate materials. Marks indicate strengths $(\oplus)$, and weaknesses $( \pm)$, and the overall evaluation of the formulation (second column). Legends: Abs. $=$ absorbed; $D R=$ dry residue

\begin{tabular}{|c|c|c|c|c|c|c|c|c|}
\hline Catalyst & Formulation & $\begin{array}{l}\text { Gelation } \\
\text { time (flasks) }\end{array}$ & $\begin{array}{l}\text { Stabilisation } \\
\text { period (within } \\
\text { stone) }\end{array}$ & $\begin{array}{l}\text { Abs. } \\
\text { product } \\
\left(\mathrm{kg} \mathrm{m}^{-2}\right)\end{array}$ & $\begin{array}{l}\text { Dry Residue } \\
\text { on stone } \\
\left(\mathrm{kg} \mathrm{m}^{-2}\right)\end{array}$ & $\begin{array}{l}\text { Drilling } \\
\text { resistance (DR) } \\
\text { increment }\end{array}$ & $\begin{array}{l}\text { Strengthening } \\
\text { depth }\end{array}$ & $\Delta E^{*}$ \\
\hline \multirow{2}{*}{$\mathrm{NH}_{3}$} & $\oplus$ 1TEOS:38EtOH: $\mathrm{NH}_{3} 0.5$ & $15 \mathrm{~d} \oplus$ & Within $7 \mathrm{~d} \oplus$ & 5.6 & 0.07 & $\sim 34 \% \oplus$ & $30 \mathrm{~mm} \oplus$ & $<3 \oplus$ \\
\hline & $\oplus$ 1TEOS:3.8EtOH: $\mathrm{NH}_{3} 0.008$ & $7 \mathrm{~d} \oplus$ & Within $7 \mathrm{~d} \oplus$ & 5.9 & 0.07 & $\sim 33 \% \oplus$ & $30 \mathrm{~mm} \oplus$ & $<3 \oplus$ \\
\hline TEOA & $\oplus$ 1TEOS:38EtOH:TEOA0.5 & $>20 \mathrm{~d} \pm$ & Within $7 \mathrm{~d} \oplus$ & 5.8 & 0.11 & $\sim 46 \% \oplus$ & $30 \mathrm{~mm} \oplus$ & $<3 \oplus$ \\
\hline
\end{tabular}

Table 6 Examples of other consolidation products (commercial and non-commercial) applied to Ançã stone, and their evaluated efficacy and risks for comparison with the consolidation effects of the silica sols produced in the present work

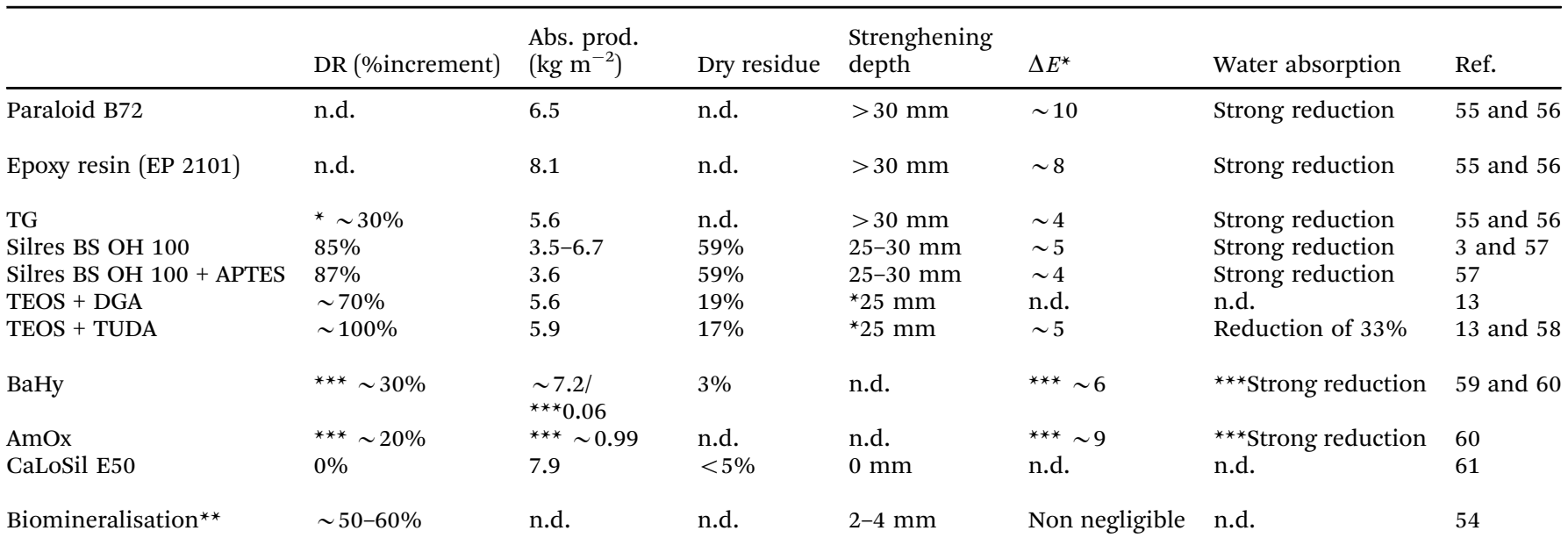

Legends: DR = drilling resistance; Abs. prod = absorbed product; ${ }^{*}$ immersion $3 \mathrm{~h} ;{ }^{* *}$ spraying (Granada method); ${ }^{* *}$ poultice, 5 h; n.d. = not determined products; TG $=$ pre-polymerised TEOS mixed with white spirit ${ }^{2}$ APTES $=3$-aminopropyltriethoxysilane; DGA = diglycolic acid; TUDA = 3,6,9-trioxaundecanedioic acid; $\mathrm{BaHy}=$ barium hydroxide; $\mathrm{AmOx}=$ ammonium oxalate; $\mathrm{CaLoSil} \mathrm{E} 50=\mathrm{Ca}(\mathrm{OH})_{2}$ nanoparticle suspension in water; Biomineralisation through the Granada method ** occurs by the 'formation of minerals by living organisms' upon 'inoculation of a specific nutritional medium to activate the resident community of carbonatogenic microbiota'. ${ }^{54}$

As can be seen in the examples presented in Table 6, the effectiveness of a treatment can often only be increased at the expense of an increase in harmfulness, something that has recently been the subject of researcher's attention. ${ }^{54}$ Commercial alkoxysilanes, other TEOS-based products, acrylic and epoxy resins usually tend to promote strength-gain dependent on: high dry residue, non-negligible colour alteration (higher risk of incompatibility $\Delta E^{*}>3^{53}$ ) and/or strong reduction of water absorption properties on the treated stone. Only some inorganic products have proved to produce small increment of strength, but also dependent on non-negligible colour alteration and/or strong reduction of water absorption properties. Newly proposed bio-consolidation options seem also need further improvement (see Table 6).

Hence, the improved sols for the consolidation of carbonate materials considered in Table 5 present potential 'effectiveness' through their increment of drilling resistance in depth, with an apparent homogeneous strengthening action. The results presented here also show the low potential 'harmfulness' concerning the formation of hardened superficial layers and colour alteration. In addition, the produced sols increased drilling resistance with a total low amount of dry residue (Table 5), compared to commercial consolidant products, such as for instance Silres BS OH 100, where a dry residue of $c a .1 .5 \mathrm{~kg} \mathrm{~m}^{-2}$ can be observed on carbonate stones. ${ }^{62}$ The largest advantage expected for such results is a low probability of blockage of the stone pores, and consequently the low effect on the water vapour permeability and similar stone properties, by affecting less or clogging the stone pores, which is an advantage in terms of consolidation.

Therefore, these results show that the most promising and viable sols produced as potential consolidants for the treated porous carbonate stones (Table 5) deserve to be further investigated. Future research work is necessary for building up a complete and pertinent assessment of compatibility and incompatibility risks on the latter sols, which include different treatment application methodologies, water permeability tests, viability on other carbonate substrates, in situ application and durability studies.

\section{Conclusions}

TEOS-based sols were catalysed with ammonia, a primary amine (Oct) and two alkanolamines (secondary, DEOA, and, tertiary, 
TEOA), aiming to explore the potential consolidation for carbonate stone materials. The roles of the different basic catalysts on the microstructure of the final gels were investigated, revealing their most relevant effects on the sol-gel mechanisms.

Overall, highly cracked and a small group of monolithic xerogels were achieved. Sols for application in calcite blends and stone samples were selected, excluding the precipitated or fast-gelling ones, which are unsuitable for consolidation actions. The incorporation of amines and alkanolamines in the sol structure induces important textural changes at the microscopic scale. As expected, depending on the type and amount of the catalysts (as well as on the ratios of the precursor/catalyst and precursor/solvent-content), which control hydrolysis and pre-condensation degrees, there were also changes in the xerogel texture and microstructure.

Cohesive monoliths of calcite powder, as well as significant and uniform drilling resistance increments caused by the treatment of the stone samples, were obtained with some of the developed sols under TEOS-basic catalysis, which revealed potential consolidation ability when applied to carbonate stones. The incorporation of $n$-octylamine in a second step reduced the gelation time and prevents silica precipitation, but is frequently responsible for the formation of a superficial hard 'crust'.

Based on the results herein disclosed, the most promising sols are: 1TEOS:38EtOH: $\mathrm{NH}_{3} 0.5$, 1TEOS:7.6EtOH: $\mathrm{NH}_{3} 0.03$, and 1TEOS:3.8EtOH: $\mathrm{NH}_{3} 0.008$; 1TEOS:38EtOH:TEOA0.5, and 1TEO$\mathrm{S}: 7.6 \mathrm{EtOH}$ :TEOA0.03. Acceptable consolidation in depth was achieved, seemingly dependent on the modifications of the silica network by the catalyst.

This study reveals the possibility of developing TEOS-based sols catalysed in basic media, which possess effective consolidation properties and high potential to be applied on carbonate stone materials.

\section{Conflicts of interest}

There are no conflicts of interest to declare.

\section{Acknowledgements}

The authors acknowledge Fundação para a Ciência e Tecnologia (FCT) for funding the Project NanoCStoneH - Innovative nanocomposite for the conservation and consolidation of carbonate stone heritage - (PTDC/ECI-EGC/29006/2017) and CQE (UIDB/00100/2020) and the support of CERIS.

\section{References}

1 G. Wheeler and E. S. Goins, Alkoxysilanes and the Consolidation of Stone, Getty Publications, 2005.

2 A. P. Ferreira Pinto and J. D. Rodrigues, J. Cult. Herit., 2008, 9, 38-53.
3 B. Sena da Fonseca, A. P. Ferreira Pinto, S. Piçarra and M. D. F. Montemor, Advanced Materials for the Conservation of Stone, 2018, 185-207.

4 A. Pintus, M. C. Aragoni, G. Carcangiu, L. Giacopetti, F. Isaia, V. Lippolis, L. Maiore, P. Meloni and M. Arca, New J. Chem., 2018, 42, 11593-11600.

5 B. Sena Da Fonseca, S. Piçarra, A. P. Ferreira Pinto, M. J. Ferreira and M. F. Montemor, New J. Chem., 2017, 41, 2458-2467.

6 M. Ban, E. Mascha, J. Weber, A. Rohatsch and J. D. Rodrigues, Materials, 2019, 12, ma12010156.

7 J. Delgado Rodrigues, Historical Constructions, 2001, 3-14.

8 C. A. Price and E. Doehne, Stone conservation: an overview of current research, Getty publications, 2010.

9 Z. Pápay and Á. Török, Engineering Geology for Society and Territory - Volume 8: Preservation of Cultural Heritage, 2015, pp. 553-556.

10 M. J. Mosquera, M. Bejarano, N. De la Rosa-Fox and L. Esquivias, J. Sol-Gel Sci. Technol., 2003, 26, 1227-1231.

11 E. K. Kim, J. Won, J. Y. Do, S. D. Kim and Y. S. Kang, J. Cult. Herit., 2009, 10, 214-221.

12 B. Sena da Fonseca, M. J. Ferreira, M. G. Taryba, S. Piçarra, A. P. Ferreira Pinto and M. D. F. Montemor, J. Cult. Herit., 2019, 37, 63-72.

13 B. Sena da Fonseca, S. Piçarra, A. P. Ferreira Pinto, M. D. F. Montemor, B. S. D. Fonseca, S. Piçarra, A. P. F. Pinto and M. D. F. Montemor, Pure Appl. Chem., 2016, 88, 1117-1128.

14 M. J. Mosquera, J. Pozo, L. Esquivias, T. Rivas and B. Silva, J. Non-Cryst. Solids, 2002, 311, 185-194.

15 C. Salazar-Hernández, R. Zárraga, S. Alonso, S. Sugita, S. Calixto and J. Cervantes, J. Sol-Gel Sci. Technol., 2009, 49, 301-310.

16 J. Cervantes, R. Zárraga and C. Salazar-Hernández, Organotin catalysts in organosilicon chemistry, 2012.

17 E. Franzoni, G. Graziani and E. Sassoni, J. Sol-Gel Sci. Technol., 2015, 74, 398-405.

18 J. Brus and P. Kotlík, Stud. Conserv., 1996, 41, 55-59.

19 M. J. Mosquera, D. M. De Los Santos, A. Montes and L. Valdez-Castro, Langmuir, 2008, 24, 2772-2778.

20 B. Sena da Fonseca, S. Piçarra, A. P. Ferreira Pinto and M. D. F. Montemor, New J. Chem., 2016, 40, 7493-7503.

21 C. Alié, F. Ferauche, R. Pirard, A. J. Lecloux and J. P. Pirard, J. Non-Cryst. Solids, 2001, 289, 88-96.

22 J. F. Illescas and M. J. Mosquera, ACS Appl. Mater. Interfaces, 2012, 4, 4259-4269.

23 F. Xu, J. Yu, D. Li, N. Xiang, Q. Zhang and L. Shao, J. Sol-Gel Sci. Technol., 2014, 71, 204-210.

24 F. Xu, H. Liu and D. Li, J. Porous Mater., 2016, 23, 1133-1141.

25 S. Nandy, D. Kundu and M. K. Naskar, J. Sol-Gel Sci. Technol., 2014, 72, 49-55.

26 D. S. Facio, M. Luna and M. J. Mosquera, Microporous Mesoporous Mater., 2017, 247, 166-176.

27 Y. Xiang, X. Li, A. Du, S. Wu, J. Shen and B. Zhou, J. Coat. Technol. Res., 2017, 14, 447-454. 
28 S. T. Selvan, C. Bullen, M. Ashokkumar and P. Mulvaney, Adv. Mater., 2001, 13, 985-988.

29 B. Sena da Fonseca, A. P. Ferreira Pinto, S. Piçarra and M. F. Montemor, J. Cult. Herit., 2020, 43, 51-63.

30 Z. A. Qiao, L. Zhang, M. Guo, Y. Liu and Q. Huo, Chem. Mater., 2009, 21, 3823-3829.

31 Sol-Gel Science, ed. C. J. Brinker and G. W. Scherer, Academic Press, San Diego, 1990, ch. 3, Hydrolysis and Condensation II: Silicates, pp. 97-234.

32 R. K. Iler, The chemistry of silica: solubility, polymerization, colloid and surface properties, and biochemistry, Wiley, New York, NY, 1979.

33 H. Bergna and W. Roberts, Colloidal Silica: Fundamentals and Applications, CRC Press, 2005.

34 G. Socrates, Infrared and Raman characteristic group frequencies. Tables and charts, 2001, p. 347.

35 S. Kurtaran and G. S. Kürkçüoğlu, Vibrational Frequencies and Structural Determination of Triethanolamine and Diethanolamine by Density Functional Theory Calculations, Proceedings of the International Workshop on New Trends in Science and Technology, Çankaya University, Ankara, Turkey, 2008.

36 M. J. Mosquera, D. M. De Los Santos and T. Rivas, Langmuir, 2010, 26, 6737-6745.

37 A. G. Prado and C. Airoldi, J. Mater. Chem., 2002, 12, 3823-3826.

38 J.-K. Hong, H.-S. Yang, M.-H. Jo, H.-H. Park and S.-Y. Choi, Thin Solid Films, 1997, 308-309, 495-500.

39 D. Sawtell, Z. A. Allah, D. W. Sheel and P. A. Martin, Proceedings of the International Symposium on Plasma Chemistry 19, Bochum, Germany, 2009.

40 M. J. Mosquera, D. M. De Los Santos, T. Rivas, P. Sanmartín and B. Silva, J. Nano Res., 2009, 8, 1-12.

41 T. I. Titova and L. S. Kosheleva, Colloids Surf., 1992, 63, 97-101.

42 M. A. Melo and C. Airoldi, Dalton Trans., 2010, 39, 10217-10227.

43 H. Zhao, Y. Xin, H. Wang, Z. Zhang and S. Liu, J. Inorg. Organomet. Polym. Mater., 2011, 21, 925-928.

44 R. Narayan, U. Y. Nayak, A. M. Raichur and S. Garg, Pharmaceutics, 2018, 10, 1-49.

45 K. Kosuge and P. S. Singh, Chem. Mater., 2001, 13, 2476-2482.
46 D. S. Facio, J. A. Ordoñez, M. L. Gil, L. A. Carrascosa and M. J. Mosquera, Coatings, 2018, 8, 170.

47 M. Mosquera, RIIPAC: Revista sobre Patrimonio Cultural, 2013, 153-172.

48 M. Bouchoucha, M. F. Côté, R. C-Gaudreault, M. A. Fortin and F. Kleitz, Chem. Mater., 2016, 28, 4243-4258.

49 J. Wang, A. Sugawara-Narutaki, M. Fukao, T. Yokoi, A. Shimojima and T. Okubo, ACS Appl. Mater. Interfaces, 2011, 3, 1538-1544.

50 E. Mine and M. Konno, J. Chem. Eng. Jpn., 2001, 34, 545-548. 51 J. R. Clifton and G. J. C. Frohnsdorff, Conservation of Historic Stone Buildings and Monuments, National Research Council, National Academy Press, Washington D. C., 1982, pp. 287-311.

52 J. F. Illescas and M. J. Mosquera, Proceedings of the 18th International Symposium on the Conservation of Monuments in the Mediterranean Basin: Monument Damage Hazards and Rehabilitation Technologies (NTUA, Univ. Patras, TEE - Sq. Western Greece, 31 May - 2 June 2010, Patras), 2010.

53 J. D. Rodrigues and A. Grossi, J. Cult. Herit., 2007, 8, 32-43. 54 J. Delgado Rodrigues and A. P. Ferreira Pinto, J. Cult. Herit., 2019, 39, 82-92.

55 A. P. Ferreira Pinto and J. Delgado Rodrigues, J. Cult. Herit., 2012, 13, 154-166.

56 A. P. Pinto and J. D. Rodrigues, Stud. Conserv., 2014, 59, 79-90.

57 A. P. Ferreira Pinto, J. Delgado Rodrigues, S. Bracci and B. Sacchi, International Symposium Stone Consolidation in Cultural Heritage Research and Preactice. Lisbon, May 2008, Proceedings, 2008, 71-79.

58 B. M. Sena da Fonseca, PhD thesis, Instituto Superior Técnico, Universidade de Lisboa, 2018.

59 J. Delgado Rodrigues and A. P. Ferreira Pinto, J. Cult. Herit., 2016, 19, 467-476.

60 S. Bracci, B. Sacchi, A. P. Ferreira Pinto and J. Delgado Rodrigues, Stone Consolidation in Cultural Heritage, 2008, pp. 81-90.

61 D. Costa and J. Delgado Rodrigues, 12th International Congress on the Deterioration and Conservation of Stone, Columbia University, New York, 2012, 2013.

62 B. Sena da Fonseca, A. P. Ferreira Pinto, S. Piçarra and M. F. Montemor, Mater. Des., 2017, 120, 10-21. 